\begin{tabular}{|c|l|}
\hline Title & Mean-Field Behavior for the Survival Probability and the Percolation Point-to-Surface Connectivity \\
\hline Author(s) & Sakai, A kira \\
\hline Citation & Journal of Statistical Physics, 117(1-2), 1111-130 \\
\hline https:/doi.org/L0.1023/B:JOSS.0000044061.83860.62 \\
\hline Issue Date & 2004_10-01 \\
\hline Doc URL & http://hdl.handle.net/2115/57817 \\
\hline Rights & The final publication is available at link.springer.com \\
\hline Type & article (author version) \\
\hline File Information & JSP_117_111-.pdf \\
\hline
\end{tabular}

Instructions for use 


\title{
Mean-field behavior for the survival probability and the percolation point-to-surface connectivity
}

\author{
Akira Sakai*
}

November 20, 2003

\begin{abstract}
We consider the critical survival probability (up to time $t$ ) for oriented percolation and the contact process, and the point-to-surface (of the ball of radius $t$ ) connectivity for critical percolation. Let $\theta_{t}$ denote both quantities. We prove in a unified fashion that, if $\theta_{t}$ exhibits a power law and both the two-point function and its restricted version exhibit the same mean-field behavior, then $\theta_{t} \asymp t^{-1}$ for the time-oriented models with $d>4$ and $\theta_{t} \asymp t^{-2}$ for percolation with $d>7$.
\end{abstract}

\section{Contents}

1 Introduction $\quad 1$

2 Models and the results $\quad 3$

2.1 Models . . . . . . . . . . . . . . . . . . . . . . . 3

2.2 Results . . . . . . . . . . . . . . . . . . . . . 5

3 Proofs $\quad 6$

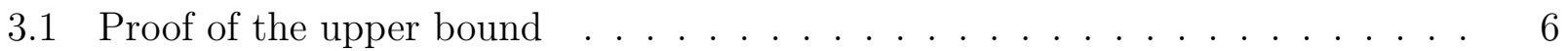

3.2 Proof of the lower bound . . . . . . . . . . . . . . . . . 8

\section{Introduction}

Percolation, oriented percolation and the contact process are known to exhibit a phase transition. Various interesting properties around the model-dependent critical point $p_{\mathrm{c}}$ have been studied and revealed, but still there are many open problems. One of the most important problems is to investigate critical exponents that characterize singular behavior of observables. Some of them were identified in certain situations.

In this paper, we consider the critical survival probability up to time $t$ for oriented percolation and the contact process, and the probability of the origin $o \in \mathbb{Z}^{d}$ being connected to the surface of the ball of radius $t$, centered at the origin, for critical percolation. Since the survival probability is a time-oriented version of the point-to-surface connectivity, we denote both quantities by $\theta_{t}$. It is believed that $\theta_{t}$ exhibits a power law: $\theta_{t} \approx t^{-1 / \delta_{\mathrm{r}}}$ as $t \rightarrow \infty$ (in some

${ }^{*}$ EURANDOM, P.O. Box 513, 5600 MB Eindhoven, The Netherlands. sakai@eurandom.tue.nl

${ }^{\dagger}$ Updated: October 12, 2004 
appropriate sense). In the percolation school, $\delta_{\mathrm{r}}$ is sometimes called the one-arm exponent. Lawler, Schramm and Werner proved $\delta_{\mathrm{r}}=48 / 5$ for the two-dimensional site percolation on the triangular lattice, using the estimates for the stochastic Loewner evolution with parameter 6 (see [21] for a precise statement). Except for this result, there has been no proof of existence of $\delta_{\mathrm{r}}$, or identification of its values for finite-range models in mathematically rigorous manner, even in high dimensions.

In contrast, the behavior of the two-point function is well-understood in high dimensions. For percolation, the two-point function at $p_{\mathrm{c}}$, denoted $\tau(x)$, is the probability of $o, x \in \mathbb{Z}^{d}$ being connected to each other, defined at $p_{\mathrm{c}}$. It has been proved that $\tau(x) \asymp|x|^{-(d-2+\eta)}$ as $|x| \rightarrow \infty$ with $\eta=0$ when $d>6$ and the number $N$ of neighbors is sufficiently large [9, 10], where

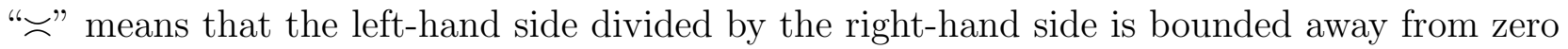
and infinity. For the time-oriented models, the two-point function at $p_{\mathrm{c}}$, denoted $\tau_{t}(x)$, is, in terms of the contact process, the probability of $x \in \mathbb{Z}^{d}$ being infected at time $t$ by the infected individual at $o \in \mathbb{Z}^{d}$ at time 0 , defined at $p_{\mathrm{c}}$. It has been proved that $\sup _{x} \tau_{t}(x) \asymp t^{-d / \alpha}$, $\hat{\tau}_{t} \equiv \sum_{x} \tau_{t}(x) \asymp t^{\eta}$ and $\sum_{x}|x|^{2} \tau_{t}(x) / \hat{\tau}_{t} \asymp t^{2 \nu}$ as $t \rightarrow \infty$, with $\alpha=2, \eta=0$ and $\nu=1 / 2$, when the spatial dimension $d$ is above 4 and $N$ is sufficiently large [17, 19, 20, 23]. These dimension-independent values of the critical exponents are equal to the values for branching random walk (the mean-field model). Let $\rho\left(\fallingdotseq 1 / \delta_{\mathrm{r}}\right)$ be defined by $\theta_{t} \asymp t^{-\rho}$ as $t \rightarrow \infty$. It is not so hard to see that $\eta=0$ implies $\rho \leq 2$ for percolation with $d>4$ and $\rho \leq 1$ for the time-oriented models with $d \geq 1$ (see Section 3.1), where the upper bounds are the mean-field values of $\rho$.

On the other hand, the critical exponents are known to satisfy the so-called hyperscaling inequalities, e.g., $d-2+\eta \geq 2 \rho$ for percolation [27] and $d \nu \geq \eta+2 \rho$ for the time-oriented models $[25,(5.2)$ and (5.4)], where the critical exponents were defined in a wider sense. Other hyperscaling inequalities were also derived in [7, 25, 27]. By those inequalities, the mean-field values are known to be incompatible with $d<6$ for percolation and with $d<4$ for the timeoriented models. These threshold dimensions are called the upper critical dimensions for the corresponding models.

In this paper, we prove in a unified way that $\rho$ takes on the mean-field values for the timeoriented models with $d>4$ and for percolation with $d>7$, if $\rho$ exists and both the two-point function and its restricted version exhibit the same mean-field behavior (see Assumption 2.1). The assumption on the restricted two-point function is expected to hold above the upper critical dimension for each model, but is still insufficient to extend $\rho=2$ for percolation down to $d>6$. For sufficiently spread-out oriented percolation with $d>4$, the asymptotic behavior of $\theta_{t}$ with $\rho=1$ will be reported in $[15,16]$, without any assumption on the restricted twopoint function. In this respect, our results are not so strong as the results in $[15,16]$ for oriented percolation. However, the approach reported in this paper is short and intuitive, and more importantly, gives a unified approach for both the time-oriented models and percolation. We expect that, with the help of the random-current representation [1], our unified approach could be applied to the single-spin expectation $\left\langle\sigma_{o}\right\rangle_{t}$ for Ising ferromagnets in the ball of radius $t$ (with plus-boundary condition), and result in the mean-field behavior, i.e., $\left\langle\sigma_{o}\right\rangle_{t} \asymp t^{-1}$ as $t \rightarrow \infty$, at the critical temperature in high dimensions. This will be discussed in [26].

We organize the rest of this paper as follows. In Section 2, we define the models and state the main result. A brief explanation of the proof is given at the end of Section 2, and the detailed proof is given in Section 3. 


\section{Models and the results}

\subsection{Models}

We consider the $d$-dimensional integer lattice $\mathbb{Z}^{d}$ as space. For $L \geq 1$, let

$$
\Omega=\left\{x \in \mathbb{Z}^{d}: 0<|x| \leq L\right\}, \quad D(x)=N^{-1} \mathbb{1}_{\{x \in \Omega\},}
$$

where $|x|$ is the Euclidean norm of $x, N$ is the cardinality of $\Omega$, and $\mathbb{1}\{\cdots\}$ is the indicator function. The model with $L=1$ is the nearest-neighbor model, where $N=2 d$. We call the model with $L>1$ the spread-out model, where $N=O\left(L^{d}\right)$ (see, e.g., [17] for a more general definition). Our models are defined in terms of $D$ as follows.

Percolation. A bond $\{x, y\}$ is an unordered pair of distinct sites in $\mathbb{Z}^{d}$, and is occupied with probability $p D(y-x)$ and vacant with probability $1-p D(y-x)$, independently of the other bonds, where $p \in[0, N]$ is the expected number of occupied bonds growing out of a single site. We denote by $\mathbb{P}_{p}$ the probability distribution for the bond variables. We say that $x$ is connected to $y$, and write $x \leftrightarrow y$, if either $x=y$ or there is a path of occupied bonds between $x$ and $y$. We define $\mathcal{C}(x)=\left\{y \in \mathbb{Z}^{d}: x \leftrightarrow y\right\}$. For $\mathcal{Z} \subset \mathbb{Z}^{d}$, we write $\{x \leftrightarrow \mathcal{Z}\}=\{\mathcal{C}(x) \cap \mathcal{Z} \neq \varnothing\}$.

It is known that there is a critical value $p_{\mathrm{c}}=p_{\mathrm{c}}(d, L) \geq 1$ such that $\sum_{x} \mathbb{P}_{p}(o \leftrightarrow x)$ is finite if and only if $p<p_{\mathrm{c}}$ and diverges as $p \uparrow p_{\mathrm{c}}$. Let

$$
\mathcal{B}_{t}=\left\{x \in \mathbb{Z}^{d}:|x| \leq t\right\}, \quad \partial \mathcal{B}_{t}=\left\{x \in \mathbb{Z}^{d}: t \leq|x| \leq t+L\right\} .
$$

and define the two-point function and the point-to-surface connectivity at $p_{\mathrm{c}}$ as

$$
\tau(x)=\mathbb{P}_{p_{\mathrm{c}}}(o \leftrightarrow x), \quad \theta_{t}=\mathbb{P}_{p_{\mathrm{c}}}\left(o \leftrightarrow \partial \mathcal{B}_{t}\right) .
$$

We are interested in the critical exponents $\eta$ and $\rho$, defined by

$$
\tau(x) \asymp\|x\|^{-(d-2+\eta)}, \quad \theta_{t} \asymp\|t\|^{-\rho},
$$

where $f \asymp g$ means that $f / g$ is bounded away from zero and infinity, and where $\|\cdot\|=|\cdot| \vee 1$. Note that $\|\cdot\|$ is not a norm on $\mathbb{R}^{d}$, but it satisfies the following properties: for $x, y \in \mathbb{R}^{d}$ and $r>0$

$$
\|x+y\| \leq\|x\|+\|y\|, \quad\|r x\| \begin{cases}\leq r\|x\|, & \text { if } r \geq 1, \\ \geq r\|x\|, & \text { if } r<1 .\end{cases}
$$

We also note that the above definition of $\rho$ is based on the assumption that $\theta_{t}$ decays as $t \rightarrow \infty$. This has been confirmed only when $d=2$ or $d \geq 19$ with $L=1$, and $d>6$ with $L \gg 1$ (see, e.g., [8, 12]).

It has been proved that $\eta=0$ for the nearest-neighbor model with $d \gg 6[9]$ and for the spread-out model with $d>6$ and $L \gg 1$ [10]. The critical exponent $\eta$ is believed to be independent of the range $L$, as long as it is finite (universality), and thus is expected to be zero for all $d>6$ and $L \geq 1$. This dimension-independent value of $\eta$ equals the corresponding value for the mean-field model. Various other critical exponents are also known to take on their respective mean-field values, if (see [3] and references therein)

$$
\nabla_{\ell} \equiv \sup _{x \notin \mathcal{B}_{\ell}}(\tau * D * \tau * \tau)(x) \rightarrow 0, \quad \text { as } \ell \rightarrow \infty,
$$

where “*” represents a convolution in $\mathbb{Z}^{d}$. With the help of $[10$, Proposition $1.7(\mathrm{i})], \eta=0$ implies $\nabla_{\ell}=O\left(\|\ell\|^{-(d-6)}\right)$ if $d>6$, and thus implies the mean-field values for all the other critical exponents, except for $\rho$ until now. 
Oriented percolation and the contact process. We begin with oriented percolation. A bond $((x, t),(y, t+1))$ is an ordered pair of sites in $\mathbb{Z}^{d} \times \mathbb{Z}_{+}$, and is occupied with probability $p D(y-x)$ and vacant with probability $1-p D(y-x)$, independently of the other bonds, where $p \in[0, N]$. We say that $(x, s)$ is connected to $(y, t)$, and write $(x, s) \rightarrow(y, t)$, if either $(x, s)=(y, t)$ or there is an oriented path of occupied bonds from $(x, s)$ to $(y, t)$. Let $\mathcal{C}(x, s)=\left\{(y, t) \in \mathbb{Z}^{d} \times \mathbb{Z}_{+}\right.$: $(x, s) \rightarrow(y, t)\}$. For $\mathcal{Z} \subset \mathbb{Z}^{d} \times \mathbb{Z}_{+}$, we define $\{(x, s) \rightarrow \mathcal{Z}\}=\{\mathcal{C}(x, s) \cap \mathcal{Z} \neq \varnothing\}$.

The contact process is a model for the spread of an infection in $\mathbb{Z}^{d}$, and is regarded as continuous-time oriented percolation in $\mathbb{Z}^{d} \times \mathbb{R}_{+}$, via the following graphical representation. Along each time line $\{x\} \times \mathbb{R}_{+}$, we place points in the manner of a Poisson process with intensity 1 , independently of the other time lines. For each ordered pair of distinct time lines from $\{x\} \times \mathbb{R}_{+}$to $\{y\} \times \mathbb{R}_{+}$, we place oriented bonds $((x, t),(y, t)), t \geq 0$, in the manner of a Poisson process with intensity $p D(y-x)$, independently of the other Poisson processes, where $p \geq 0$ is the infection rate. We say that $(x, s)$ is connected to $(y, t)$, and write $(x, s) \rightarrow(y, t)$, if either $(x, s)=(y, t)$ or there is an oriented path in $\mathbb{Z}^{d} \times \mathbb{R}_{+}$from $(x, s)$ to $(y, t)$ using the Poisson bonds and time-line segments traversed in the increasing-time direction without traversing the Poisson points. We define $\mathcal{C}(x, s)$ and $\{(x, s) \rightarrow \mathcal{Z}\}$ for $\mathcal{Z} \subset \mathbb{Z}^{d} \times \mathbb{R}_{+}$similarly to oriented percolation.

We denote by $\mathbb{P}_{p}$ the probability distributions for these time-oriented models. It is known that there is a critical value $p_{\mathrm{c}}=p_{\mathrm{c}}(d, L) \geq 1$, depending on the models, such that the sum over $t \in \mathbb{Z}_{+}$of $\sum_{x} \mathbb{P}_{p}((o, 0) \rightarrow(x, t))$ for oriented percolation, or the integral of $\sum_{x} \mathbb{P}_{p}((o, 0) \rightarrow$ $(x, t))$ with respect to $t \in \mathbb{R}_{+}$for the contact process, is finite if and only if $p<p_{\mathrm{c}}$ and diverges as $p \uparrow p_{\text {c }}$. Let

$$
\mathcal{B}_{t}=\mathbb{Z}^{d} \times[0, t], \quad \partial \mathcal{B}_{t}=\mathbb{Z}^{d} \times\{t\},
$$

and define the two-point function and the survival probability at $p_{\mathrm{c}}$ as

$$
\tau_{t}(x)=\mathbb{P}_{p_{\mathrm{c}}}((o, 0) \rightarrow(x, t)), \quad \theta_{t}=\mathbb{P}_{p_{\mathrm{c}}}\left((o, 0) \rightarrow \partial \mathcal{B}_{t}\right) .
$$

We are interested in the critical exponents $\alpha, \eta, \nu$ and $\rho$, defined by

$$
\begin{aligned}
\bar{\tau}_{t} \equiv \sup _{x \in \mathbb{Z}^{d}} \tau_{t}(x) \asymp\|t\|^{-d / \alpha}, & \hat{\tau}_{t} \equiv \sum_{x \in \mathbb{Z}^{d}} \tau_{t}(x) \asymp\|t\|^{\eta}, \\
\sum_{x \in \mathbb{Z}^{d}}|x|^{2} \frac{\tau_{t}(x)}{\hat{\tau}_{t}} \asymp\|t\|^{2 \nu}, & \theta_{t} \asymp\|t\|^{-\rho},
\end{aligned}
$$

where, by analogy, we used the same letters $\eta$ and $\rho$ for the critical exponents of the spatial sum of the two-point function and the survival probability, respectively.

It has been proved that $(\alpha, \eta, \nu)=\left(2,0, \frac{1}{2}\right)$ for the time-oriented models with $d>4$ and $L \gg 1[17,20]$. The same result except for $\alpha=2$ was proved in [23] for nearest-neighbor oriented percolation with $d \gg 4$, but there have been no results on this set of exponents for the nearest-neighbor contact process. Other critical exponents for both the nearest-neighbor and spread-out time-oriented models are known to take on their respective mean-field values, if (see [4] and references therein)

$$
\nabla_{\ell} \equiv \sup _{\substack{x:|x| \geq \ell \\ t \geq 0}} \nabla(x, t) \rightarrow 0, \quad \text { as } \ell \rightarrow \infty
$$

where, for oriented percolation,

$$
\nabla(x, t)=\sum_{\substack{s, s^{\prime} \in \mathbb{Z}_{+}: \\ t \leq s^{\prime} \leq s}} \sum_{y \in \mathbb{Z}^{d}} \tau_{s+1}(y)\left(\tau_{s-s^{\prime}} * D * \tau_{s^{\prime}-t}\right)(y-x),
$$


and for the contact process,

$$
\nabla(x, t)=\int_{t}^{\infty} d s \int_{t}^{s} d s^{\prime} \sum_{y \in \mathbb{Z}^{d}} \tau_{s}(y)\left(\tau_{s-s^{\prime}} * D * \tau_{s^{\prime}-t}\right)(y-x) .
$$

Since the range of the set of infected sites almost surely grows at most linearly [5], $(\alpha, \eta)=$ $(2,0)$ implies $\nabla_{\ell}=O\left(\|\ell\|^{-(d-4) / 2}\right)$ if $d>4$, and thus implies the mean-field values for all the other critical exponents than $\rho$.

\section{$2.2 \quad$ Results}

In this paper, we prove in a unified fashion for all three models that the mean-field behavior for the two-point function implies the mean-field values of $\rho$, assuming existence of $\rho$ and the following assumption.

Assumption 2.1. There are positive constants $C_{1}=C_{1}(d, L)$ and $C_{2}=C_{2}(d, L)$ that are independent of $t$ such that, for the time-oriented models,

$$
\sum_{(x, s) \in \mathcal{B}_{t / 2}} \mathbb{P}_{p_{\mathrm{c}}}\left((o, 0) \rightarrow(x, s),(o, 0) \nrightarrow \partial \mathcal{B}_{t}\right) \geq C_{1}\|t\|,
$$

and for percolation,

$$
\sum_{x \in \mathcal{B}_{t / 2+L}} \mathbb{P}_{p_{\mathrm{c}}}\left(o \leftrightarrow x, o \leftrightarrow \partial \mathcal{B}_{t}\right) \geq C_{2}\|t\|^{2},
$$

where $\mathcal{B}_{t / 2+L}=\mathcal{B}_{t / 2} \cup \partial \mathcal{B}_{t / 2}$.

If $\eta=0$, then we obtain $\sum_{s \leq t / 2} \hat{\tau}_{s} \asymp\|t\|$ for the time-oriented models with $d \geq 1$ and $\sum_{x \in \mathcal{B}_{t / 2+L}} \tau(x) \asymp\|t\|^{2}$ for percolation with $d \geq 2$. Therefore, Assumption 2.1 states, in a weak sense, that the above restricted two-point functions exhibit the same mean-field behavior as the unrestricted two-point functions.

Theorem 2.2. Suppose that $\eta=0$ and $\alpha=2$ (the latter is only for the time-oriented models). If $\rho$ exists and Assumption 2.1 holds, then $\rho=1$ for the time-oriented models with $d>4$ and $\rho=2$ for percolation with $d>7$.

We briefly explain the main idea of the proof. It is easy to show that $\eta=0$ implies $\rho \leq 1$ for the time-oriented models with $d \geq 1$ and $\rho \leq 2$ for percolation with $d>4$ (see Section 3.1). It thus suffices to prove the opposite inequalities for $\rho$. Let us consider typical configurations for $\theta_{t}$. When $t \gg 1$, there may be a pivotal bond for the connection from the origin to the boundary $\partial \mathcal{B}_{t}$. We take notice of the last pivotal bond $b$, where we have a connection from the origin to the first endpoint of $b$ and two disjoint connections from the second endpoint of $b$ to $\partial \mathcal{B}_{t}$ (see Figure 1). If we could bound the probability of these configurations from below by $\theta_{t}^{2}$ times the sum of the unrestricted two-point function (over $b=\left(\underline{b}, \bar{b}\right.$ ) with $\bar{b} \in \mathcal{B}_{t / 2}$, as in Figure 1), then $\eta=0$ implies

$$
t^{-\rho} \geq \begin{cases}c t^{1-2 \rho}, & \text { for the time-oriented models } \\ c t^{2-2 \rho}, & \text { for percolation }\end{cases}
$$

for some positive constant $c$, and thus $\rho \geq 1$ for the time-oriented models and $\rho \geq 2$ for percolation. 

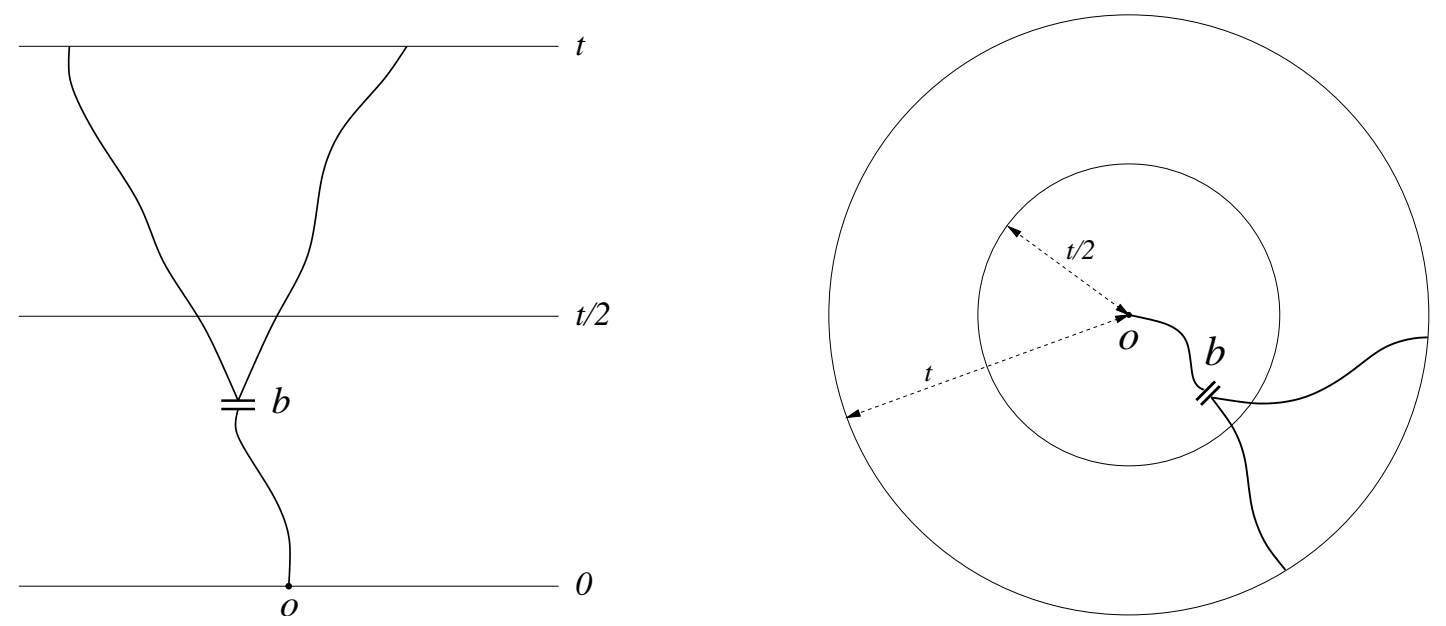

Figure 1: Typical configurations for $\theta_{t}$.

To realize the above idea, we have to control the correction. As we will show in Section 3.2, most error terms can be made small by letting $\nabla_{\ell} \ll 1$ and $t \gg 1$ in high dimensions. However, the correction due to the above approximation using the unrestricted two-point function cannot be controlled by a finite number of applications of the BK inequality (see, e.g., [6, 8]), and here we will use Assumption 2.1. The desired asymptotic behavior of $\theta_{t}$ for spread-out oriented percolation with $d>4$ and $L \gg 1$ will be reported in $[15,16]$, with no assumption on the restricted two-point function. The proof in $[15,16]$ is based on the lace expansion for $\theta_{t}$, and the difference between the restricted and unrestricted two-point functions is efficiently taken into account along the expansion. Our proof of Theorem 2.2 does not depend on the full expansion as in $[15,16]$, and Assumption 2.1 is inevitable.

We remark that Assumption 2.1 is still insufficient to fully control the boundary effect and thus to obtain $\rho=2$ for percolation with $d>6$. To improve the result down to $d>6$, we may also need some information on the restricted two-point function close to the boundary (see Remark at the end of Section 3.2).

\section{Proofs}

We prove Theorem 2.2 in two steps. First, in Section 3.1, we prove that $\eta=0$ implies $\rho \leq 1$ for the time-oriented models with $d \geq 1$ and $\rho \leq 2$ for percolation with $d>4$. Then, in Section 3.2, we prove that $\eta=0$ and $\alpha=2$ (the latter is only for the time-oriented models) imply the opposite inequalities for $\rho$, if $d>4$ for the time-oriented models and $d>7$ for percolation, assuming existence of $\rho$ and Assumption 2.1.

In the rest of this paper, we omit the subscript $p_{\mathrm{c}}$ and write $\mathbb{E}$ for the expectation with respect to $\mathbb{P}=\mathbb{P}_{p_{\mathrm{c}}}$. We will use $c$ to denote a finite positive constant which may depend on $d$ and $L$, but whose exact value is unimportant and may change from line to line.

\subsection{Proof of the upper bound}

Proof for the time-oriented models. Let

$$
I_{t}=\mathbb{1}\left\{(o, 0) \rightarrow \partial \mathcal{B}_{t}\right\}, \quad X_{t}=\sum_{x \in \mathbb{Z}^{d}} \mathbb{1}\{(o, 0) \rightarrow(x, t)\}
$$


so that $\mathbb{E}\left(I_{t}\right)=\theta_{t}$ and $\mathbb{E}\left(X_{t}\right)=\hat{\tau}_{t}$. By the Schwarz inequality, we obtain

$$
\hat{\tau}_{t}^{2}=\mathbb{E}\left(I_{t} X_{t}\right)^{2} \leq \mathbb{E}\left(I_{t}^{2}\right) \mathbb{E}\left(X_{t}^{2}\right)=\theta_{t} \sum_{x, y} \mathbb{P}((o, 0) \rightarrow(x, t),(o, 0) \rightarrow(y, t)) .
$$

If $(o, 0) \rightarrow(x, t)$ and $(o, 0) \rightarrow(y, t)$ occur simultaneously, then there exists a $(z, s) \in \mathcal{B}_{t}$ such that $(o, 0) \rightarrow(z, s)$ occurs and that $(z, s) \rightarrow(x, t)$ and $(z, s) \rightarrow(y, t)$ occur disjointly, i.e., on disjoint sets of bonds. Using the Markov property, the BK inequality and $\eta=0$, we can bound the sum in (3.2) by

$$
\int_{0}^{t} d s \sum_{x, y, z \in \mathbb{Z}^{d}} \tau_{s}(z) \tau_{t-s}(x-z) \tau_{t-s}(y-z)=\int_{0}^{t} d s \hat{\tau}_{s} \hat{\tau}_{t-s}^{2} \leq c\|t\| .
$$

(The integral is replaced by $\sum_{s=0}^{t}$ for oriented percolation.) Together with (3.2), we thus obtain $\rho \leq 1$, if $\rho$ exists.

Remark. For spread-out oriented percolation with $d>4$ and $L \gg 1$, Theorem 4.1 and Lemma 4.2 in [14] imply that the left-hand side of (3.2) is asymptotically $A^{2}$, while the sum in the right-hand side of (3.2) is asymptotically $A^{3} V t$, where $A$ and $V$ are constants depending only on $d$ and $L$. This leads to a lower bound on $\theta_{t}$ like $(A V t)^{-1}$, which is consistent with [14, Theorem 1.5], where the $\operatorname{limit}_{t \rightarrow \infty} t \theta_{t}$, if it exists, equals $2(A V)^{-1}$.

Proof for percolation. We follow the same strategy as above. Let

$$
I_{t}=\mathbb{1}_{\left\{o \leftrightarrow \partial \mathcal{B}_{t}\right\}}, \quad X_{t}=\sum_{x \in \partial \mathcal{B}_{t}} \mathbb{1}_{\{o \leftrightarrow x\}}
$$

Using the Schwarz inequality as in (3.2), we obtain

$$
\left[\sum_{x \in \partial \mathcal{B}_{t}} \tau(x)\right]^{2}=\mathbb{E}\left(I_{t} X_{t}\right)^{2} \leq \mathbb{E}\left(I_{t}^{2}\right) \mathbb{E}\left(X_{t}^{2}\right)=\theta_{t} \sum_{x, y \in \partial \mathcal{B}_{t}} \mathbb{P}(o \leftrightarrow x, o \leftrightarrow y)
$$

Since $\eta=0$, the leftmost quantity is bounded from below by $c\|t\|^{2}$. If $o \leftrightarrow x$ and $o \leftrightarrow y$ occur simultaneously, then there is a $z \in \mathbb{Z}^{d}$ such that $o \leftrightarrow z, z \leftrightarrow x$ and $z \leftrightarrow y$ occur disjointly. By the BK inequality and $\eta=0$, the sum in the right-hand side of (3.5) is bounded by

$$
\begin{gathered}
\sum_{\substack{x, y \in \partial \mathcal{B}_{t} \\
z \in \mathbb{Z}^{d}}} \tau(z) \tau(x-z) \tau(y-z)=\sum_{\substack{x, y \in \partial \mathcal{B}_{t} \\
z \in \mathcal{B}_{t / 2}}} \tau(z) \tau(x-z) \tau(y-z)+\sum_{\substack{x, y \in \partial \mathcal{B}_{t} \\
z \notin \mathcal{B}_{t / 2}}} \tau(z) \tau(x-z) \tau(y-z) \\
\leq c\|t\|\left\|^{2(2-d)+2(d-1)} \sum_{z \in \mathcal{B}_{t / 2}}\right\| z\left\|^{2-d}+c\right\| t\left\|^{2-d} \sum_{\substack{x, y \in \partial \mathcal{B}_{t} \\
z \in \mathbb{Z}^{d}}}\right\| x-z\left\|^{2-d}\right\| y-z \|^{2-d}
\end{gathered}
$$

where we used $|x-z| \geq t / 2$ and $|y-z| \geq t / 2$ in the first sum, and $|z| \geq t / 2$ in the second sum. By [10, Proposition 1.7(i)], the convolution of $\|x-z\|^{2-d}$ and $\|y-z\|^{2-d}$ is bounded by $c\|x-y\|^{4-d}$ if $d>4$, whose sum over $x, y \in \partial \mathcal{B}_{t}$ is bounded by $c\|t\|^{2(d-1)+4-d}=c\|t\|^{d+2}$. Therefore, (3.1) is bounded by $c\|t\|^{4}$, and we obtain $\rho \leq 2$ using (3.5). 


\subsection{Proof of the lower bound}

In this section, we will use $\epsilon=\epsilon(\rho)$ defined by

$$
\epsilon(\rho) \begin{cases}>0 \text { (but arbitrarily small), } & \text { if } \rho=1, \\ =0, & \text { if } \rho \neq 1\end{cases}
$$

for both the time-oriented models and percolation.

Proof for the time-oriented models. We only consider oriented percolation, since the same idea given below also applies to the time-discretized contact process in $[17,24]$ that weakly converges to the original contact process as the discretized-time unit tends to zero. We prove below

$$
\theta_{t} \geq c\left[1-O(\bar{\nabla})-O\left(\|t\|^{-(d-4) / 2+\epsilon}\right)\right]\|t\|^{1-2 \rho},
$$

and thus prove Theorem 2.2 for the time-oriented models, assuming $\bar{\nabla} \equiv \sup _{x} \nabla(x, 0) \ll 1$. In the proof of (3.8), we will require $p_{\mathrm{c}} \leq 3 / 2$, which is a consequence of $\bar{\nabla} \ll 1$, if $d>4$ $[18,22,24]$. We will also assume existence of a constant $a>1$, which is independent of $d$ and $L$, such that $\sum_{s \leq t / 2} \hat{\tau}_{s} \leq a C_{1}\|t\|\left(\right.$ cf., (2.14)) and $K \leq \theta_{t}\|t\|^{\rho} \leq a K$ for some $K>0$, which may depend on $\bar{d}$ and $L$. After the proof, we briefly discuss how to remove all these extra assumptions.

The survival probability $\theta_{t}$ is the probability of the event that there is a path of occupied bonds from $(o, 0)$ to $\partial \mathcal{B}_{t}$. This event can be decomposed into two disjoint events depending on whether or not $(o, 0)$ is doubly connected to $\partial \mathcal{B}_{t}$, denoted by $(o, 0) \rightrightarrows \partial \mathcal{B}_{t}$, which means that there are at least two bond-disjoint occupied paths from $(o, 0)$ to $\partial \mathcal{B}_{t}$. If $(o, 0)$ is connected but not doubly connected to $\partial \mathcal{B}_{t}$, then there is an occupied pivotal bond $b=(\underline{b}, \bar{b})$ for $(o, 0) \rightarrow \partial \mathcal{B}_{t}$ such that $(o, 0) \rightarrow \underline{b}, \bar{b} \rightrightarrows \partial \mathcal{B}_{t}$ and $\mathcal{C}^{b}(o, 0) \cap \partial \mathcal{B}_{t}=\varnothing$, where $\mathcal{C}^{b}(o, 0)$ is the set of sites in $\mathbb{Z}^{d} \times \mathbb{Z}_{+}$connected from $(o, 0)$ without using $b$. Restricting the location of $\bar{b}$ to $\mathcal{B}_{t / 2}$ gives

$$
\theta_{t} \geq \sum_{b: \bar{b} \in \mathcal{B}_{t / 2}} \frac{1}{N} \mathbb{P}\left((o, 0) \rightarrow \underline{b}, \bar{b} \rightrightarrows \partial \mathcal{B}_{t}, \mathcal{C}^{b}(o, 0) \cap \partial \mathcal{B}_{t}=\varnothing\right)
$$

where we used $p_{\mathrm{c}} \geq 1$ and the fact that the status of $b$ is independent of the event in $\mathbb{P}$. For an event $E$ and $\mathcal{Z} \subset \mathbb{Z}^{d} \times \mathbb{Z}_{+}$, let $\{E$ in $\mathcal{Z}\}$ be the set of bond configurations whose restriction on bonds $b$ contained in $\mathcal{Z}$ (i.e., both $\underline{b}$ and $\bar{b}$ are in $\mathcal{Z}$ ) are in $E$. Using this notion, we can rewrite the probability in the right-hand side of (3.9) as (see [13, Definition 2.2(c) and Lemma 2.5])

$$
\mathbb{P}\left(\left\{(o, 0) \rightarrow \underline{b}, \mathcal{C}^{b}(o, 0) \cap \partial \mathcal{B}_{t}=\varnothing\right\} \text { in } \mathcal{C}^{b}(o, 0),\left\{\bar{b} \rightrightarrows \partial \mathcal{B}_{t}\right\} \text { in } \mathcal{C}^{b}(o, 0)^{\mathrm{c}}\right) .
$$

By the "conditioning over clusters" technique [2, 12, 13], (3.10) equals

$$
\begin{aligned}
\mathbb{E}( & \left.\mathbb{1}_{\left\{(o, 0) \rightarrow \underline{b}, \mathcal{C}^{b}(o, 0) \cap \partial \mathcal{B}_{t}=\varnothing\right\}} \mathbb{P}\left(\bar{b} \rightrightarrows \partial \mathcal{B}_{t} \text { in } \mathcal{C}^{b}(o, 0)^{\mathrm{c}}\right)\right) \\
= & \mathbb{P}\left((o, 0) \rightarrow \underline{b}, \mathcal{C}^{b}(o, 0) \cap \partial \mathcal{B}_{t}=\varnothing\right) \mathbb{P}\left(\bar{b} \rightrightarrows \partial \mathcal{B}_{t}\right) \\
& \quad-\mathbb{E}\left(\mathbb{1}_{\left\{(o, 0) \rightarrow \underline{b}, \mathcal{C}^{b}(o, 0) \cap \partial \mathcal{B}_{t}=\varnothing\right\}}\left[\mathbb{P}\left(\bar{b} \rightrightarrows \partial \mathcal{B}_{t}\right)-\mathbb{P}\left(\bar{b} \rightrightarrows \partial \mathcal{B}_{t} \text { in } \mathcal{C}^{b}(o, 0)^{\mathrm{c}}\right)\right]\right)
\end{aligned}
$$

In the first term, we use $\left\{\mathcal{C}^{b}(o, 0) \cap \partial \mathcal{B}_{t}=\varnothing\right\} \supset\left\{(o, 0) \not \supset \partial \mathcal{B}_{t}\right\}$ and $\mathbb{P}\left(\bar{b} \rightrightarrows \partial \mathcal{B}_{t}\right) \geq \mathbb{P}((o, 0) \rightrightarrows$ $\partial \mathcal{B}_{t}$ ), and apply the definition of $D$ in (2.1) and Assumption 2.1. Then, we obtain

$$
\begin{aligned}
\theta_{t} \geq & C_{1}\|t\| \mathbb{P}\left((o, 0) \rightrightarrows \partial \mathcal{B}_{t}\right) \\
& -\sum_{b: \bar{b} \in \mathcal{B}_{t / 2}} \frac{1}{N} \mathbb{E}\left(\mathbb{1}\left\{(o, 0) \rightarrow \underline{b}, \mathcal{C}^{b}(o, 0) \cap \partial \mathcal{B}_{t}=\varnothing\right\} \mathbb{P}\left(\left\{\bar{b} \rightrightarrows \partial \mathcal{B}_{t}\right\} \backslash\left\{\bar{b} \rightrightarrows \partial \mathcal{B}_{t} \text { in } \mathcal{C}^{b}(o, 0)^{\mathrm{c}}\right\}\right)\right) .
\end{aligned}
$$


This is the starting point for the proof of (3.8).

First, we consider the first term in the right-hand side of (3.12), and show that

$$
C_{1}\|t\| \mathbb{P}\left((o, 0) \rightrightarrows \partial \mathcal{B}_{t}\right) \geq \frac{C_{1} K^{2}}{4 e}\left(1-4^{\rho+3} a^{2} \bar{\nabla}-c\|t\|^{-(d-4) / 2+\epsilon}\right)\|t\|^{1-2 \rho}
$$

For the proof of this inequality, it suffices to investigate $\mathbb{P}\left((o, 0) \rightrightarrows \partial \mathcal{B}_{t}\right)$. Restricting the number of occupied bonds growing out of $(o, 0)$ to two, we obtain

$$
\mathbb{P}\left((o, 0) \rightrightarrows \partial \mathcal{B}_{t}\right) \geq\left(\frac{p_{\mathrm{c}}}{N}\right)^{2}\left(1-\frac{p_{\mathrm{c}}}{N}\right)^{N-2} \sum_{\langle x, y\rangle} \mathbb{P}\left(\begin{array}{c}
(x, 1) \rightarrow \partial \mathcal{B}_{t},(y, 1) \rightarrow \partial \mathcal{B}_{t}, \\
\mathcal{C}(x, 1) \cap \mathcal{C}(y, 1)=\varnothing \text { in } \mathcal{B}_{t}
\end{array}\right)
$$

where $\sum_{\langle x, y\rangle}$ is the sum over all pairs of distinct sites in $\Omega$. By conditioning over the cluster $\mathcal{C}(x, 1)$, the right-hand side of (3.14) equals

$$
\left(\frac{p_{\mathrm{c}}}{N}\right)^{2}\left(1-\frac{p_{\mathrm{c}}}{N}\right)^{N-2} \sum_{\langle x, y\rangle} \mathbb{E}\left(\mathbb{1}_{\left\{(x, 1) \rightarrow \partial \mathcal{B}_{t}\right\}} \mathbb{P}\left((y, 1) \rightarrow \partial \mathcal{B}_{t} \text { in } \mathcal{C}(x, 1)^{\mathrm{c}}\right)\right) .
$$

Note that $p_{\mathrm{c}}^{2}\left(1-\frac{p_{\mathrm{c}}}{N}\right)^{N-2}$ is bounded from above by $36 e^{-3 / 2}$ using $p_{\mathrm{c}} \geq 1$, while it is bounded from below by $e^{-1}$ using $p_{\mathrm{c}} \leq 3 / 2$. Therefore, if we ignore the condition "in $\mathcal{C}(x, 1)^{\mathrm{c}}$ " in $(3.15)$, then we obtain the lower bound $\frac{e^{-1}}{N^{2}}\left(\begin{array}{c}N \\ 2\end{array}\right) \theta_{t}^{2} \geq \frac{K^{2}}{4 e}\|t\|^{-2 \rho}$, which is the main contribution in the right-hand side of (3.13). The correction for this is

$$
\left(\frac{p_{\mathrm{c}}}{N}\right)^{2}\left(1-\frac{p_{\mathrm{c}}}{N}\right)^{N-2} \sum_{\langle x, y\rangle} \mathbb{E}\left(\mathbb{1}_{\left\{(x, 1) \rightarrow \partial \mathcal{B}_{t}\right\}} \mathbb{P}\left(\left\{(y, 1) \rightarrow \partial \mathcal{B}_{t}\right\} \backslash\left\{(y, 1) \rightarrow \partial \mathcal{B}_{t} \text { in } \mathcal{C}(x, 1)^{\mathrm{c}}\right\}\right)\right) .
$$

To complete the proof of (3.13), we bound (3.16) from above. Since the event inside $\mathbb{P}$ in (3.16) is the event that all occupied paths from $(y, 1)$ to $\partial \mathcal{B}_{t}$ go through $\mathcal{C}(x, 1)$, there must be a $(z, s) \in \mathcal{C}(x, 1)$ such that $(y, 1) \rightarrow(z, s) \rightarrow \partial \mathcal{B}_{t}$. By the Markov property, the expectation in (3.16) is bounded by

$$
\begin{aligned}
& \mathbb{E}\left(\mathbb{1}\left\{(x, 1) \rightarrow \partial \mathcal{B}_{t}\right\}\right. \\
&\left.\quad \sum_{(z, s) \in \mathcal{C}(x, 1)} \tau_{s-1}(z-y) \theta_{t-s}\right) \\
&=\sum_{(z, s)} \mathbb{P}\left((x, 1) \rightarrow \partial \mathcal{B}_{t},(z, s) \in \mathcal{C}(x, 1)\right) \tau_{s-1}(z-y) \theta_{t-s}
\end{aligned}
$$

We separate the sum over $s$ into $\sum_{s \leq t / 2}$ and $\sum_{s>t / 2}$.

1. For $\sum_{s \leq t / 2}$, we use the fact that there is a site $\left(z^{\prime}, s^{\prime}\right)$ such that $(x, 1) \rightarrow\left(z^{\prime}, s^{\prime}\right) \rightarrow \partial \mathcal{B}_{t}$ and $\left(z^{\prime}, s^{\prime}\right) \rightarrow(z, s)$ occur disjointly. By the BK inequality, the contribution to (3.17) is bounded by

$$
\sum_{s=2}^{t / 2} \sum_{s^{\prime}=1}^{s} \sum_{z, z^{\prime} \in \mathbb{Z}^{d}} \tau_{s^{\prime}-1}\left(z^{\prime}-x\right) \tau_{s-s^{\prime}}\left(z-z^{\prime}\right) \tau_{s-1}(z-y) \theta_{t-s} \theta_{t-s^{\prime}}
$$

where $s \geq 2$, because $x \neq y$. Using $t-s^{\prime} \geq t-s \geq t / 2$ and (2.5), we bound (3.18) by $4^{\rho}(a K)^{2} \bar{\nabla}\|t\|^{-2 \rho}$. This gives the second term in the right-hand side of (3.13), where we used $\left(\frac{p_{\mathrm{c}}}{N}\right)^{2}\left(1-\frac{p_{\mathrm{c}}}{N}\right)^{N-2}\left(\begin{array}{c}N \\ 2\end{array}\right) \leq 18 e^{-3 / 2}<4^{2} e^{-1}$. 
2. For $\sum_{s>t / 2}$, we use (3.17) instead of (3.18). By ignoring the event $\left\{(x, 1) \rightarrow \partial \mathcal{B}_{t}\right\},(3.17)$ is bounded by

$$
\sum_{s=t / 2}^{t} \hat{\tau}_{s-1} \bar{\tau}_{s-1} \theta_{t-s} \leq c\|t\|^{-d / 2} \sum_{s=t / 2}^{t}\|t-s\|^{-\rho} .
$$

Since $\rho \leq 1$, the right-hand side is bounded by $c\|t\|^{-d / 2+1-\rho+\epsilon} \leq c\|t\|^{-2 \rho-(d-4) / 2+\epsilon}$, which gives the third term in the right-hand side of (3.13).

This completes the proof of (3.13).

Next, we investigate the second term in (3.12), and prove that

$$
\begin{gathered}
\sum_{b: \bar{b} \in \mathcal{B}_{t / 2}} \frac{1}{N} \mathbb{E}\left(\mathbb{1}\left\{(o, 0) \rightarrow \underline{b}, \mathcal{C}^{b}(o, 0) \cap \partial \mathcal{B}_{t}=\varnothing\right\} \mathbb{P}\left(\left\{\bar{b} \rightrightarrows \partial \mathcal{B}_{t}\right\} \backslash\left\{\bar{b} \rightrightarrows \partial \mathcal{B}_{t} \text { in } \mathcal{C}^{b}(o, 0)^{c}\right\}\right)\right) \\
\leq\left(8^{\rho} a^{3} C_{1} K^{2} \bar{\nabla}+c\|t\|^{-(d-4) / 2+\epsilon}\right)\|t\|^{1-2 \rho} .
\end{gathered}
$$

Note that the event $\left\{\bar{b} \rightrightarrows \partial \mathcal{B}_{t}\right\} \backslash\left\{\bar{b} \rightrightarrows \partial \mathcal{B}_{t}\right.$ in $\left.\mathcal{C}^{b}(o, 0)^{\mathrm{c}}\right\}$ implies existence of a $(z, s) \in \mathcal{C}^{b}(o, 0)$ such that $\bar{b} \rightarrow(z, s) \rightarrow \partial \mathcal{B}_{t}$ and $\bar{b} \rightarrow \partial \mathcal{B}_{t}$ occur disjointly. Using the BK inequality, we can extract $\tau_{s-s^{\prime}}(z-v) \theta_{t-s}$ and $\theta_{t-s^{\prime}}$, where $\bar{b}=\left(v, s^{\prime}\right)$. Using $(2.1)$, we bound the left-hand side of (3.20) by

$$
\begin{aligned}
& \sum_{\substack{(z, s),\left(v, s^{\prime}\right): \\
1 \leq s^{\prime}<t / 2}} \sum_{b: \bar{b}=\left(v, s^{\prime}\right)} \frac{1}{N} \mathbb{P}\left((o, 0) \rightarrow \underline{b},(z, s) \in \mathcal{C}^{b}(o, 0)\right) \tau_{s-s^{\prime}}(z-v) \theta_{t-s} \theta_{t-s^{\prime}} \\
& \leq \sum_{\substack{(z, s),\left(v, s^{\prime}\right): \\
1 \leq s^{\prime}<t / 2}} \sum_{\substack{(y, r),\left(u, s^{\prime}-1\right) \\
0 \leq r<s^{\prime}}} \tau_{r}(y) \tau_{s^{\prime}-1-r}(u-y) D(v-u) \tau_{s-s^{\prime}}(z-v) \tau_{s-r}(z-y) \theta_{t-s} \theta_{t-s^{\prime}} \\
& \leq \frac{2^{\rho} a K}{\|t\| \rho} \sum_{r=0}^{t / 2-1} \hat{\tau}_{r} \sum_{s=r+1}^{t} \sum_{s^{\prime}=r+1}^{(t / 2) \wedge s} \sum_{x \in \mathbb{Z}^{d}}\left(\tau_{s^{\prime}-1-r} * D * \tau_{s-s^{\prime}}\right)(x) \tau_{s-r}(x) \theta_{t-s}
\end{aligned}
$$

where $(y, r) \in \mathbb{Z}^{d} \times \mathbb{Z}_{+}$in the second expression is a branch point arising from the intersection $\{(o, 0) \rightarrow \underline{b}\} \cap\left\{(z, s) \in \mathcal{C}^{b}(o, 0)\right\}$, and we used $s^{\prime} \leq t / 2$ and $(2.5)$ to bound $\theta_{t-s^{\prime}}$ as in the last inequality. We separate the sum over $s$ into $\sum_{s \leq 3 t / 4}$ and $\sum_{s>3 t / 4}$.

1. For $\sum_{s \leq 3 t / 4}$, we bound $\theta_{t-s}$ by $4^{\rho} a K\|t\|^{-\rho}$ using (2.5), and then bound the remaining term by $\bar{\nabla} \sum_{r=0}^{t / 2-1} \hat{\tau}_{r} \leq a C_{1} \bar{\nabla}\|t\|$. This gives the first term in the right-hand side of (3.20).

2. For $\sum_{s>3 t / 4}$, we bound $\bar{\tau}_{s-r}$ by $c\|t\|^{-d / 2}$ using $r<t / 2$, and then bound the remaining term, using $\rho \leq 1$, by

$$
c\|t\| \sum_{r=0}^{t / 2-1} \sum_{s=3 t / 4}^{t}\|t-s\|^{-\rho} \leq c\|t\|^{3-\rho+\epsilon} .
$$

This gives the second term in the right-hand side of (3.20).

This completes the proof of (3.20).

The proof of (3.8) is completed by (3.13) and (3.20). We obtain (2.16) from (3.8) if $\bar{\nabla} \ll 1$, $t \gg 1$ and $d>4$. Together with $\rho \leq 1$ proved in Section 3.1, this completes the proof of $\rho=1$. 
Remark. In the above proof, we exploited the assumptions stated below (3.8). These assumptions can be removed via a delocalization argument [4] (or, it is also called ultraviolet regularization $[2,3,12])$. In fact, we can prove that there is a $c_{\ell}>0$ such that

$$
t^{-\rho} \geq c_{\ell}\left[1-O\left(\nabla_{\ell}\right)-O\left(t^{-(d-4) / 2+\epsilon}\right)\right] t^{1-2 \rho}, \quad \text { for } t \gg \ell
$$

Recall that $(\alpha, \eta)=(2,0)$ implies $\lim _{\ell \rightarrow \infty} \nabla_{\ell}=0$, as explained below (2.13). Taking $\ell$ and $t$ in (3.23) sufficiently large, independently of $d$ and $L$, we obtain (2.16) for the time-oriented models. Therefore, we do not need to control the constants in terms of $d$ and $L$, as in the extra assumptions stated below (3.8).

We briefly explain the idea for the proof of (3.23). Recall (3.9), where $b$ is the last pivotal bond for $(o, 0) \rightarrow \partial \mathcal{B}_{t}$. The space-time rectangle $\mathcal{R}_{\ell}(b)$ is defined as

$$
\mathcal{R}_{\ell}(b)=\left\{\underline{b}+\left(r e_{b}, s\right) \in \mathbb{Z}^{d} \times \mathbb{Z}_{+}: r \in[-\ell, \ell], s \in[0, \ell]\right\},
$$

where $e_{b}=(v-u) /|v-u|$ for $b=((u, s),(v, s+1))$. We may modify the occupation status of bonds contained in $\mathcal{R}_{\ell}(b)$, in order to thin the connection from $(o, 0)$ to $\partial \mathcal{B}_{t}$. Let $E_{\mathcal{R}_{\ell}(b)}$ be such an event that $\underline{b}$ is "minimally" connected, via $b$, to both $X_{ \pm} \equiv \underline{b}+\left( \pm \ell e_{b}, \ell\right)$. Then, we obtain (cf., (3.9))

$$
\theta_{t} \geq \sum_{b: \bar{b} \in \mathcal{B}_{t / 2}} \mathbb{P}\left(E_{\mathcal{R}_{\ell}(b)}\right) \mathbb{P}\left(\begin{array}{c}
(o, 0) \rightarrow \underline{b}, \mathcal{C}^{\mathcal{R}_{\ell}(b)}(o, 0) \cap \partial \mathcal{B}_{t}=\varnothing, \\
\left\{X_{+} \rightarrow \partial \mathcal{B}_{t}\right\} \circ\left\{X_{-} \rightarrow \partial \mathcal{B}_{t}\right\}
\end{array}\right)
$$

where $E_{1} \circ E_{2}$ is the event that $E_{1}$ and $E_{2}$ occur disjointly, and $\mathcal{C}^{\mathcal{R}_{\ell}(b)}(o, 0)$ is the set of sites connected from $(o, 0)$ without using any bonds contained in $\mathcal{R}_{\ell}(b)$. In $(3.25)$, we used the fact that $E_{\mathcal{R}_{\ell}(b)}$ is independent of the other three events in $\mathbb{P}$. We choose $c_{\ell}=\inf _{b} \mathbb{P}\left(E_{\mathcal{R}_{\ell}(b)}\right)$. For the remaining term, we follow the same strategy as in the proof for the case $\bar{\nabla} \ll 1$, except that we do not need an argument around (3.14), which is also a sort of delocalization argument. This leads to (3.23).

It remains to determine $E_{\mathcal{R}_{\ell}(b)}$. This was well-explained in [4] for the time-discretized contact process. A variant of $E_{\mathcal{R}_{\ell}(b)}$ in [4] was chosen in such a way that $c_{\ell}$ is bounded away from zero uniformly in the discretized-time unit. It is not hard to adapt the idea of [4] to our settings, and we refrain from giving its details. See [4, Figure 1].

Proof for percolation. The strategy is the same as above. We prove below

$$
\theta_{t} \geq c\left[1-O\left(\nabla_{0}\right)-O\left(\|t\|^{-(d-5-\rho \vee 1)+\epsilon}\right)\right]\|t\|^{2-2 \rho},
$$

for $t \geq 2 L$ (so that $\partial \mathcal{B}_{t / 2} \subset \mathcal{B}_{t}$ ), and hence Theorem 2.2 for percolation, assuming $\nabla_{0} \ll 1$. Similarly to the proof for the time-oriented models, we will also assume that $p_{\mathrm{c}} \leq 3 / 2$, which is true when $\nabla_{0} \ll 1$ and $d>6[11,18]$, and that there is a $(d, L)$-independent constant $a>1$ such that $\sum_{x \in \mathcal{B}_{3 t / 2+L}} \tau(x) \leq a C_{2}\|t\|^{2}$ (cf., (2.15)) and $K \leq \theta_{t}\|t\|^{\rho} \leq a K$ for some $K>0$, which may depend on $d$ and $L$. These assumptions can be removed as discussed in the above remark and as in $[2,3,12]$, and we omit its details for simplicity.

The percolation version of (3.12) is

$$
\begin{aligned}
\theta_{t} \geq & C_{2}\|t\|^{2} \mathbb{P}\left(o \Leftrightarrow \partial \mathcal{B}_{3 t / 2}\right) \\
& -\sum_{b: \bar{b} \in \mathcal{B}_{t / 2}} \frac{1}{N} \mathbb{E}\left(\mathbb{1}\left\{o \leftrightarrow \underline{b}, \mathcal{C}^{b}(o) \cap \partial \mathcal{B}_{t}=\varnothing\right\} \mathbb{P}\left(\left\{\bar{b} \Leftrightarrow \partial \mathcal{B}_{t}\right\} \backslash\left\{\bar{b} \Leftrightarrow \partial \mathcal{B}_{t} \text { in } \mathcal{C}^{b}(o)^{\mathrm{c}}\right\}\right)\right),
\end{aligned}
$$


where " $\Leftrightarrow$ " represents a double connection for percolation, and we used $\mathbb{P}\left(\bar{b} \Leftrightarrow \partial \mathcal{B}_{t}\right) \geq \mathbb{P}(o \Leftrightarrow$ $\partial \mathcal{B}_{3 t / 2}$ ) and Assumption 2.1. We only discuss below bounding the first term of the right-hand side of (3.27). The second term can be estimated similarly, following the same strategy as explained below (3.20).

To investigate the first term of the right-hand side of (3.27), it thus suffices to consider $\mathbb{P}\left(o \Leftrightarrow \partial \mathcal{B}_{3 t / 2}\right)$. To bound this from below, we restrict the number of occupied bonds growing out of $o \in \mathbb{Z}^{d}$ to two. Let $\tilde{\mathcal{C}}_{3 t / 2}(x) \subset \mathcal{B}_{3 t / 2+L}$ be the set of sites to which there is an occupied path from $x$ that includes at most one bond growing from $\partial \mathcal{B}_{3 t / 2}$ and no bonds growing from $o \in \mathbb{Z}^{d}$. Then, we have (cf., (3.14))

$$
\mathbb{P}\left(o \Leftrightarrow \partial \mathcal{B}_{3 t / 2}\right) \geq\left(\frac{p_{\mathrm{c}}}{N}\right)^{2}\left(1-\frac{p_{\mathrm{c}}}{N}\right)^{N-2} \sum_{\langle x, y\rangle} \mathbb{P}\left(\begin{array}{c}
x \leftrightarrow \partial \mathcal{B}_{3 t / 2} \text { in }\{o\}^{\mathrm{c}}, y \leftrightarrow \partial \mathcal{B}_{3 t / 2} \text { in }\{o\}^{\mathrm{c}}, \\
\tilde{\mathcal{C}}_{3 t / 2}(x) \cap \tilde{\mathcal{C}}_{3 t / 2}(y)=\varnothing
\end{array}\right) .
$$

By conditioning over the cluster $\tilde{\mathcal{C}}_{3 t / 2}(x)$, the above expression equals

$$
\begin{aligned}
& \left(\frac{p_{\mathrm{c}}}{N}\right)^{2}\left(1-\frac{p_{\mathrm{c}}}{N}\right)^{N-2} \sum_{\langle x, y\rangle} \mathbb{E}\left(\mathbb{1}\left\{x \leftrightarrow \partial \mathcal{B}_{3 t / 2} \text { in }\{o\}^{\mathrm{c}}\right\} \mathbb{P}\left(y \leftrightarrow \partial \mathcal{B}_{3 t / 2} \text { in }\{o\}^{\mathrm{c}} \cap \tilde{\mathcal{C}}_{3 t / 2}(x)^{\mathrm{c}}\right)\right) \\
& =\left(\frac{p_{\mathrm{c}}}{N}\right)^{2}\left(1-\frac{p_{\mathrm{c}}}{N}\right)^{N-2} \sum_{\langle x, y\rangle}\left[\mathbb{P}\left(x \leftrightarrow \partial \mathcal{B}_{3 t / 2} \text { in }\{o\}^{\mathrm{c}}\right) \mathbb{P}\left(y \leftrightarrow \partial \mathcal{B}_{3 t / 2} \text { in }\{o\}^{\mathrm{c}}\right)\right. \\
& \left.-\mathbb{E}\left(\mathbb{1}\left\{x \leftrightarrow \partial \mathcal{B}_{3 t / 2} \text { in }\{o\}^{\mathrm{c}}\right\} \mathbb{P}\left(\left\{y \leftrightarrow \partial \mathcal{B}_{3 t / 2} \text { in }\{o\}^{\mathrm{c}}\right\} \backslash\left\{y \leftrightarrow \partial \mathcal{B}_{3 t / 2} \text { in }\{o\}^{\mathrm{c}} \cap \tilde{\mathcal{C}}_{3 t / 2}(x)^{\mathrm{c}}\right\}\right)\right)\right] .
\end{aligned}
$$

Here, we have $\mathbb{P}\left(x \leftrightarrow \partial \mathcal{B}_{3 t / 2}\right.$ in $\left.\{o\}^{\mathrm{c}}\right)$, instead of $\mathbb{P}\left(x \leftrightarrow \partial \mathcal{B}_{3 t / 2}\right)$ as explained below (3.15) for the time-oriented models. The correction is the probability of the event that all occupied paths between $x$ and $\partial \mathcal{B}_{3 t / 2}$ go through the origin, and thus is bounded by the probability of the event that $x \leftrightarrow o$ and $o \leftrightarrow \partial \mathcal{B}_{3 t / 2}$ occur disjointly. Using the BK inequality and monotonicity, we obtain

$$
\mathbb{P}\left(x \leftrightarrow \partial \mathcal{B}_{3 t / 2} \text { in }\{o\}^{\mathrm{c}}\right) \geq \mathbb{P}\left(x \leftrightarrow \partial \mathcal{B}_{3 t / 2}\right)-\tau(x) \theta_{3 t / 2} \geq \theta_{3 t / 2+L}-\tau(x) \theta_{3 t / 2} .
$$

First, we consider the contribution to (3.29) from $\mathbb{P}\left(x \leftrightarrow \partial \mathcal{B}_{3 t / 2}\right.$ in $\left.\{o\}^{\mathrm{c}}\right) \mathbb{P}\left(y \leftrightarrow \partial \mathcal{B}_{3 t / 2}\right.$ in $\left.\{o\}^{\mathrm{c}}\right)$. By $(3.30)$, the main contribution comes from $\theta_{3 t / 2+L}^{2}$ that is bounded from below by $\frac{K^{2}}{4 e}\left\|\frac{3}{2} t+L\right\|^{-2 \rho} \geq\left(4^{\rho+1} e\right)^{-1} K^{2}\|t\|^{-2 \rho}$, where we used $p_{\mathrm{c}} \leq 3 / 2$ (cf., the argument below (3.15)) and $t \geq 2 L$ together with (2.5). The contribution from the terms containing $\tau(x) \theta_{3 t / 2}$ or $\tau(y) \theta_{3 t / 2}$ is bounded by $K^{2} O\left(\nabla_{0}\right)\|t\|^{-2 \rho}$, using $N^{-2}=D(x) D(y)$ in (3.29).

Next, we investigate the contribution from the expectation in the right-hand side of (3.29). Since the event inside $\mathbb{P}$ is the event that all occupied paths from $y$ to $\partial \mathcal{B}_{3 t / 2}$ in $\{o\}^{\mathrm{c}}$ go through $\tilde{\mathcal{C}}_{3 t / 2}(x) \subset \mathcal{B}_{3 t / 2+L}$, there must be a $z \in \tilde{\mathcal{C}}_{3 t / 2}(x)$ such that $y \leftrightarrow z$ and $z \leftrightarrow \partial \mathcal{B}_{3 t / 2}$ occur disjointly. Therefore, the expectation in the right-hand side of (3.29) is bounded, using the BK inequality, by

$$
\begin{aligned}
& \mathbb{E}\left(\mathbb{1}\left\{x \leftrightarrow \partial \mathcal{B}_{3 t / 2} \text { in }\{o\}^{\mathrm{c}}\right\} \sum_{z \in \tilde{\mathcal{C}}_{3 t / 2}(x)} \tau(z-y) \mathbb{P}\left(z \leftrightarrow \partial \mathcal{B}_{3 t / 2}\right)\right) \\
& \quad \leq \sum_{z \in \mathcal{B}_{3 t / 2+L}} \mathbb{P}\left(x \leftrightarrow \partial \mathcal{B}_{3 t / 2}, z \in \tilde{\mathcal{C}}_{3 t / 2}(x)\right) \tau(z-y) \mathbb{P}\left(z \leftrightarrow \partial \mathcal{B}_{3 t / 2}\right)
\end{aligned}
$$

We separate the sum into $\sum_{z \in \mathcal{B}_{3 t / 2+L} \backslash \mathcal{B}_{t / 2}}$ and $\sum_{z \in \mathcal{B}_{t / 2}}$. 
1. For $\sum_{z \in \mathcal{B}_{3 t / 2+L} \backslash \mathcal{B}_{t / 2}}$, we ignore ${ }^{1}$ the event $\left\{x \leftrightarrow \partial \mathcal{B}_{3 t / 2}\right\}$ as in $(3.19)$, and use $\mathbb{P}(z \leftrightarrow$ $\left.\partial \mathcal{B}_{3 t / 2}\right) \leq \theta_{(3 t / 2-|z|) \vee 0}$. Then, the contribution to (3.31) is bounded by

$$
\begin{aligned}
\sum_{z \in \mathcal{B}_{3 t / 2+L} \backslash \mathcal{B}_{t / 2}} \tau(z-x) \tau(z-y) \theta_{(3 t / 2-|z|) \vee 0} & \leq c\|t\|^{(d-1)+2(2-d)}\left(L+\sum_{s=0}^{t}\|s\|^{-\rho}\right) \\
& \leq c\|t\|^{-2 \rho-(d-\rho-3-\rho \vee 1)+\epsilon}
\end{aligned}
$$

which is bounded by $c\|t\|^{-2 \rho-(d-5-\rho \vee 1)+\epsilon}$, using $\rho \leq 2$.

2. For $\sum_{z \in \mathcal{B}_{t / 2}}$, we bound $\mathbb{P}\left(z \leftrightarrow \partial \mathcal{B}_{3 t / 2}\right)$ by $a K\|t\|^{-\rho}$, and note that the event inside the other $\mathbb{P}$ in (3.31) implies existence of $w \in \mathcal{B}_{3 t / 2+L}$ such that $x \leftrightarrow w, w \leftrightarrow z$ and $w \leftrightarrow \partial \mathcal{B}_{3 t / 2}$ occur disjointly. Again by the BK inequality, the contribution to (3.31) is bounded by

$$
a K\|t\|^{-\rho} \sum_{\substack{z \in \mathcal{B}_{t / 2} \\ w \in \mathcal{B}_{3 t / 2+L}}} \tau(x-w) \tau(w-z) \tau(z-y) \mathbb{P}\left(w \leftrightarrow \partial \mathcal{B}_{3 t / 2}\right)
$$

We further separate the sum over $w$ into $\sum_{w \in \mathcal{B}_{t / 2}}$ and $\sum_{w \in \mathcal{B}_{3 t / 2+L} \backslash \mathcal{B}_{t / 2}}$.

(a) For $\sum_{w \in \mathcal{B}_{t / 2}}$, we bound $\mathbb{P}\left(w \leftrightarrow \partial \mathcal{B}_{3 t / 2}\right)$ by $a K\|t\|^{-\rho}$, and then bound the remaining term, using $x \neq y$, by $a K\|t\|^{-\rho}(\tau * \tau * \tau)(y-x) \leq a K \nabla_{0}\|t\|^{-\rho}$.

(b) For $\sum_{w \in \mathcal{B}_{3 t / 2+L} \backslash \mathcal{B}_{t / 2}}$, we use $\mathbb{P}\left(w \leftrightarrow \partial \mathcal{B}_{3 t / 2}\right) \leq \theta_{(3 t / 2-|w|) \vee 0}$ and perform the sum over $z \in \mathbb{Z}^{d}$ using [10, Proposition 1.7(i)]. Since $x, y \in \Omega$, the contribution to (3.33) is bounded by

$$
\begin{aligned}
& c\|t\|^{-\rho} \sum_{w \in \mathcal{B}_{3 t / 2+L} \backslash \mathcal{B}_{t / 2}}\||w|-L\|^{(2-d)+(4-d)}\left\|\frac{3}{2} t-|w|\right\|^{-\rho} \\
& \leq c\|t\|^{-\rho+(6-2 d)+(d-1)}\left(L+\sum_{s=0}^{t}\|s\|^{-\rho}\right) \leq c\|t\|^{-2 \rho-(d-5-\rho \vee 1)+\epsilon} .
\end{aligned}
$$

Summarizing the above estimates, we conclude that the contribution from the expectation in the right-hand side of (3.29) is bounded from above by $\left(a^{2} K^{2} \nabla_{0}+c\|t\|^{-(d-5-\rho \vee 1)+\epsilon}\right)\|t\|^{-2 \rho}$. Together with the discussion below (3.30), we complete bounding $C_{2}\|t\|^{2} \mathbb{P}\left(o \Leftrightarrow \partial \mathcal{B}_{3 t / 2}\right)$ in (3.27) from below by the same formula in the right-hand side of (3.26), where $c=O\left(C_{2} K^{2}\right)$. The sum in (3.27) can be estimated similarly to (3.33), where $z$ in (3.33) corresponds to $\bar{b}$ in (3.27). This completes the proof of (3.26).

We obtain (2.16) from (3.26) if $\nabla_{0} \ll 1, t \gg 1$ and $d>5+\rho \vee 1$, and thus obtain $\rho=2$ for $d>7$. This completes the proof.

\footnotetext{
${ }^{1}$ Some readers might wonder whether the event $\left\{x \leftrightarrow \partial \mathcal{B}_{3 t / 2}\right\}$ could be used to have less power in (3.32). In fact, if we use the inequality

$$
\mathbb{P}\left(x \leftrightarrow \partial \mathcal{B}_{3 t / 2}, z \in \tilde{\mathcal{C}}_{3 t / 2}(x)\right) \leq \sum_{w \in \mathcal{B}_{3 t / 2+L}} \tau(w-x) \tau(z-w) \theta_{(3 t / 2-|w|) \vee 0},
$$

then the contribution due to $w \in \mathcal{B}_{t / 2}$ is bounded by (3.34), while the contribution from $w \in \mathcal{B}_{3 t / 2+L} \backslash \mathcal{B}_{t / 2}$ has a worse bound $c\|t\|^{-2 \rho+\mu}$, where $\mu$ is negative only when $d>9$.
} 
Remark. The value of $\rho$ for percolation is expected to be 2 as soon as $d>6$. The main obstacle to going down from $d>7$ is in (3.32) and (3.34), which correspond respectively to (3.19) and (3.22) for the time-oriented models. In (3.19) and (3.22), the sum over $s$ is fully controlled using $\theta_{t-s} \asymp\|t-s\|^{-\rho}$. On the other hand, the point-to-surface connectivity $\theta_{(3 t / 2-|v|) \vee 0}$, with $v=z$ in (3.32) and $v=w$ in (3.34), is insufficient to obtain the desired bound, when $v$ is close to the boundary $\partial \mathcal{B}_{3 t / 2}$. This difficulty is considered to be caused by naively bounding the probability inside $\mathbb{E}$ in (3.29) as in (3.31). Since $\left\{y \leftrightarrow \partial \mathcal{B}_{3 t / 2}\right.$ in $\left.\{o\}^{\mathrm{c}}\right\} \backslash\{y \leftrightarrow$ $\partial \mathcal{B}_{3 t / 2}$ in $\left.\{o\}^{\mathrm{c}} \cap \tilde{\mathcal{C}}_{3 t / 2}(x)^{\mathrm{c}}\right\}$ is the event that all occupied paths from $y$ to $\partial \mathcal{B}_{3 t / 2}\left(\right.$ in $\left.\{o\}^{\mathrm{c}}\right)$ have to go through $\tilde{\mathcal{C}}_{3 t / 2}(x)$ before reaching to the boundary, the approximation by the unrestricted two-point function $\tau(z-y)$ in (3.31) could be very crude when $z$ is close to $\partial \mathcal{B}_{3 t / 2}$, due to the isotropic property for percolation. If we assume that there is a $\kappa \geq 1$ such that, for $|z|=\ell$,

$$
\mathbb{P}\left(o \leftrightarrow z, o \nLeftarrow \partial \mathcal{B}_{t}\right) \leq c\|\ell\|^{2-d-\kappa}(\|\ell\| \wedge\|t-\ell\|)^{\kappa},
$$

then we will be able to obtain the desired inequality (2.16) down to $d>6$. Note that (3.35) contains the factor $\|t-\ell\|$ that decreases as $z$ approaches the boundary $\partial \mathcal{B}_{t}$, that the sum of the right-hand side over $z \in \mathcal{B}_{t}$ is bounded by $c\|t\|^{2}$, and that the limit $t \rightarrow \infty$ of the right-hand side, while $\ell$ or $\ell / t$ is fixed, is $c\|\ell\|^{2-d}$. Therefore, (3.35) is a good candidate for the bound on the restricted two-point function, though we have not proved whether (3.35) really holds or does not. (The random walk restricted Green's function satisfies (3.35) at $\ell=t$ with $\kappa=1$.)

\section{Acknowledgements}

I am grateful to Federico Camia and Remco van der Hofstad for many valuable comments on the first draft of this paper. I would also like to thank Antal Járai and Frank den Hollander for stimulating discussions, and Takashi Hara for his unpublished manuscript. This work was supported by a Postdoctoral Fellowship of EURANDOM, the Netherlands.

\section{References}

[1] M. Aizenman. Geometric analysis of $\phi^{4}$ fields and Ising models. Commun. Math. Phys. 86 (1982): 1-48.

[2] M. Aizenman and C. M. Newman. Tree graph inequalities and critical behavior in percolation models. J. Statist. Phys. 36 (1984): 107-143.

[3] D. J. Barsky and M. Aizenman. Percolation critical exponents under the triangle condition. Ann. Probab. 19 (1991): 1520-1536.

[4] D. J. Barsky and C. C. Wu. Critical exponents for the contact process under the triangle condition. J. Statist. Phys. 91 (1998): 95-124.

[5] C. Bezuidenhout and G. Grimmett. The critical contact process dies out. Ann. Probab. 18 (1990): 1462-1482.

[6] C. Bezuidenhout and G. Grimmett. Exponential decay for subcritical contact and percolation processes. Ann. Probab. 19 (1991): 984-1009.

[7] J. T. Chayes and L. Chayes. On the upper critical dimension of Bernoulli percolation. Commun. Math. Phys. 113 (1987): 27-48. 
[8] G. Grimmett. Percolation. Springer, Berlin (1999).

[9] T. Hara. Critical two-point functions for nearest-neighbour high-dimensional self-avoiding walk and percolation. Unpublished manuscript.

[10] T. Hara, R. van der Hofstad and G. Slade. Critical two-point functions and the lace expansion for spread-out high-dimensional percolation and related models. Ann. Probab. 31 (2003): 349-408.

[11] T. Hara and G. Slade. Mean-field critical behaviour for percolation in high dimensions. Commun. Math. Phys. 128 (1990): 333-391.

[12] T. Hara and G. Slade. Mean-field behaviour and the lace expansion. Probability and Phase Transition (ed. G. R. Grimmett). Kluwer, Dordrecht (1994): 87-122.

[13] T. Hara and G. Slade. The scaling limit of the incipient infinite cluster in high-dimensional percolation. I. Critical exponents. J. Statist. Phys. 99 (2000): 1075-1168.

[14] R. van der Hofstad, F. den Hollander and G. Slade. Construction of the incipient infinite cluster for spread-out oriented percolation above $4+1$ dimensions. Commun. Math. Phys. 231 (2002): 435-461.

[15] R. van der Hofstad, F. den Hollander and G. Slade. The survival probability for critical spread-out oriented percolation above $4+1$ dimensions. I. Induction. In preparation.

[16] R. van der Hofstad, F. den Hollander and G. Slade. The survival probability for critical spread-out oriented percolation above $4+1$ dimensions. II. Expansion. In preparation.

[17] R. van der Hofstad and A. Sakai. Gaussian scaling limit for the critical spread-out contact process above the upper critical dimension. Preprint.

[18] R. van der Hofstad and A. Sakai. Critical points for spread-out self-avoiding walk, percolation and the contact process above the upper critical dimensions. Preprint.

[19] R. van der Hofstad and G. Slade. A generalised inductive approach to the lace expansion. Probab. Theory Rel. Fields 122 (2002): 389-430.

[20] R. van der Hofstad and G. Slade. Convergence of critical oriented percolation to superBrownian motion above $4+1$ dimensions. Ann. Inst. H. Poincaré Probab. Statist. 39 (2003): 413-485.

[21] G. F. Lawler, O. Schramm and W. Werner. One-arm exponent for critical 2D percolation. Electronic J. Probab. 7 (2002). Paper no. 2.

[22] B. G. Nguyen and W.-S. Yang. Triangle condition for oriented percolation in high dimensions. Ann. Probab. 21 (1993): 1809-1844.

[23] B. G. Nguyen and W.-S. Yang. Gaussian limit for critical oriented percolation in high dimensions. J. Statist. Phys. 78 (1995): 841-876.

[24] A. Sakai. Mean-field critical behavior for the contact process. J. Statist. Phys. 104 (2001): 111-143.

[25] A. Sakai. Hyperscaling inequalities for the contact process and oriented percolation. J. Statist. Phys. 106 (2002): 201-211. 
[26] A. Sakai. Mean-field behavior for the finite-volume single-spin expectation for Ising ferromagnets. In preparation.

[27] H. Tasaki. Hyperscaling inequalities for percolation. Commun. Math. Phys. 113 (1987): $49-65$. 